\title{
PREVALÊNCIA DE SINAIS E SINTOMAS AVALIADOS EM UM GRUPO DE EMAGRECIMENTO DE UM CENTRO UNIVERSITÁRIO DO OESTE DO PARANÁ
}

\section{PREVALENCE OF SIGNS AND SYMPTOMS EVALUATED IN A WEIGHT LOSS GROUP AT A UNIVERSITY CENTER IN WESTERN PARANÁ}

\author{
Larissa de Carvalho Zimmermann ${ }^{1}$, Thais Mariotto Cezar ${ }^{2}$ \\ ${ }^{1}$ Nutricionista, Centro Universitário Fundação Assis Gurgacz (FAG). ${ }^{2}$ Nutricionista Mestre, Docente \\ do Curso de Nutrição, Centro Universitário Fundação Assis Gurgacz (FAG). \\ * Autor correspondente: thamariotto@hotmail.com , https://orcid.org/0000-0001-8954-6584
}

DOI: $10.35984 / f j h . v 2 i 2.159$

\section{RESUMO}

Sabemos que nos dias atuais os hábitos alimentares da população estão cada vez mais inadequados, as pessoas procuram por praticidade e não por qualidade de nutrientes, sendo assim a alimentação inadequada tem interferido na qualidade de vida da população e o acompanhamento nutricional é imprescindível para a pessoa ter uma qualidade de vida. Rastreamento metabólico é um questionário que foi elaborado pelo Instituto Brasileiro de Nutrição Funcional (IBNF). É utilizado para investigar variados sinais e sintomas, contexto que o paciente dará sua pontuação de acordo com o que está sentindo nos últimos dias de acordo com a tabela de escala de pontos. No final do questionário, esses pontos são somados e interpretados de acordo com a tabela de resultados do IBNF. O objetivo do estudo foi verificar melhora de sinais e sintomas em grupos de emagrecimento composto por colaboradores de um centro universitário do Oeste do Paraná. O grupo contou com 8 participantes, todas do sexo feminino, com idades entre 18 e 60 anos. As participantes passaram por avaliação nutricional e foram acompanhadas por dez semanas. Nesse período, estas participaram de palestras, dinâmicas e conversas em grupos. Nos encontros semanais foram abordados assuntos pertinentes que colaborariam com uma melhora nos hábitos alimentares, com 0 objetivo de emagrecimento. No primeiro encontro, as participantes responderam três questionários, entre eles o Questionário de Rastreamento Metabólico (QRM). Após a análise da somatória dos resultados, foi possível verificar e interpretar os dados podendo observar a melhora de $48,29 \%$ dos sinais e sintomas, uma vez que no primeiro encontro a somatória total de 5 participantes foi $>40$ indicativos de absoluta certeza de hipersensibilidade e de 3 participantes foi $>100$ indicativos de que a pessoa está com a saúde muito ruim. Já no décimo encontro podemos verificar que a somatória total de 5 participantes foi $<30$ indicando que a pessoa está com menos sinais e sintomas, de 2 pessoas foram $>40$ indicativos de de hipersensibilidade e de 1 pessoa foi $>100$ indicativos de que a pessoa está com a saúde muito ruim. Pode-se observar que houve melhora significativa nos sinais e sintomas das participantes quando submetidas a um acompanhamento nutricional. Deste modo, é notório que o acompanhamento nutricional é imprescindível para promoção e prevenção de doenças. Palavras chave: Nutrição, hábitos alimentares, saúde.

\begin{abstract}
We know that nowadays the population's eating habits are increasingly inadequate, people look for practicality and not for quality of nutrients, so inadequate food has interfered in the quality of life of the population and nutritional monitoring is essential for the person have a good quality of life. Metabolic Tracking is a questionnaire that was prepared by the Brazilian
\end{abstract}


Institute of Functional Nutrition (BIFN). It is used to investigate various signs and symptoms, the context in which the patient will give his score according to what he is feeling in the last days according to the point scale table. At the end of the questionnaire, these points are added and interpreted according to the results table of (BIFN). The objective of the study was to verify improvements in signs and symptoms in weight loss groups composed of employees from a university center in Western Paraná. The group had 8 participants, all female, aged between 18 and 60 years. Participants underwent nutritional assessment and were followed up for ten weeks. During this period, they participated in lectures, dynamics and group conversations. In weekly meetings, pertinent subjects were addressed that would collaborate with an improvement in eating habits, with the objective of losing weight. At the first meeting, the participants answered three questionnaires, including the Metabolic Tracking Questionnaire (MTQ). After analyzing the sum of the results, it was possible to verify and interpret the data and observe an improvement of $48.29 \%$ of the signs and symptoms, since in the first meeting the total sum of 5 participants was $>40$ indicative of absolute certainty of hypersensitivity and of 3 participants it was> 100 indications that the person is in very bad health. In the tenth meeting, we can see that the total sum of 5 participants was $<30$ indicating that the person has fewer signs and symptoms, 2 people were $>40$ indicating absolute certainty of hypersensitivity and 1 person was> 100 indicating that the person is in very bad health. It can be observed that there was a significant improvement in the signs and symptoms of the participants when submitted to nutritional monitoring. Thus, it is well known that nutritional monitoring is essential for the promotion and prevention of diseases.

Keywords: Nutrition, Eating Habits, Health

\section{INTRODUÇÃO}

O comportamento alimentar está diretamente ligado ao estilo e hábitos de vida das pessoas, também é possível observar que, cada vez mais, estes são inadequados à população e têm trazido razões para o surgimento de doenças. O comportamento alimentar é de caráter muito complexo e vem sendo formado ao longo dos anos, podendo defini-lo como a maneira de se alimentar, fatores como condições financeiras, horários e companhia para realizar as refeições. Também se destaca que refeições com poucos nutrientes e lanches rápidos são os principais motivos de mudanças de comportamentos e hábitos alimentares (FRANÇA et al,2012 e VAZ et al, 2018).

Observa-se que nos dias atuais a população tem apresentado hábitos alimentares restritos em nutrientes importantes para atender às necessidades típicas da idade com predisposição a trocas de refeições. O aumento do consumo de açúcares e gorduras saturadas e a diminuição da ingestão de micronutrientes, através da alimentação, tornam a população vulnerável do ponto de vista nutricional, agregado à ausência de atividade física, este contexto favorece o surgimento de carências nutricionais e de doenças metabólicas associadas ao excesso de peso, sendo necessário o nutricionista ajudar as pessoas a modificarem seus hábitos alimentares, através da assistência nutricional (SILVA, et al, 2018).

As carências nutricionais são também mascaradas por sinais e sintomas, como cólicas, dores de cabeça, dores musculares, sangramentos que levam a exaustão e debilidade, e baixa sensibilidade visual, dificultando os níveis de concentração e produtividade. Esses sintomas podem ser observados através do questionário de rastreamento metabólico que foi elaborado pelo Instituto Brasileiro de Nutrição Funcional. É possível investigar variados sinais e sintomas dos quais o próprio paciente dará sua pontuação, avaliando de acordo com o que está sentindo nos últimos 30 dias, última semana ou últimas 48 horas, e no final, esses pontos são 
somados e utilizados como parâmetro para iniciar um rastreamento de prováveis deficiências nutricionais, hipersensibilidades, intolerâncias e alergias alimentares (GALDINO et al., 2016; MARTINEZ, 2011). Para a melhora da qualidade de vida, o acompanhamento nutricional é imprescindível, sendo utilizado com pacientes que têm o objetivo da perda de peso, mudança de estilo de vida e melhora da qualidade de vida. Estes acompanhamentos são feitos frequentemente, utilizando a avaliação antropométrica, com o intuito de avaliar como anda o processo de emagrecimento e a eficácia do plano alimentar (TIRAPEGUI et al, 2011 e RIBEIRO, 2009). Diante do exposto, o objetivo deste trabalho foi avaliar sinais e sintomas de colaboradoras da instituição por meio do Questionário de Rastreamento Metabólico que foi respondido pelos participantes no primeiro e último encontro.

\section{METODOLOGIA}

Trata-se de um estudo qualitativo e descritivo, onde se considera que há uma relação dinâmica favorável entre o mundo real e o sujeito. Não requer o uso de métodos e técnicas estatísticas e o ambiente natural é a fonte direta para coleta de dados. O processo da pesquisa e os seus resultados são os focos principais de abordagem (GIL, 2008). O projeto foi encaminhado ao Comitê de Ética em Pesquisa pela Plataforma Brasil, e após apreciação, foi considerado aprovado pelo parecer número 3.079.664. O Termo de Consentimento Livre e Esclarecido (TCLE) foi entregue e explicado ao participante do grupo e estes assinaram aceitando participar da pesquisa. O critério para participação do grupo era ter idade entre 18 e 60 anos.

O estudo foi realizado em um Centro Universitário do Oeste do Paraná, contando com 8 (oito) colaboradores. Os encontros em grupo foram realizados em uma sala de reuniões na instituição. Foram realizados encontros semanais por um período de 10 semanas. Foram ministrados, nos encontros semanais, assuntos pertinentes que colaborariam com uma melhora nos hábitos alimentares, e através da proposta de perda de peso, mudanças dos hábitos e estilo de vida, atingindo o objetivo de emagrecimento. Vários assuntos foram abordados como: saúde do intestino; contagem de calorias, qualidade dos alimentos; rotulagem de alimentos; fome e saciedade; insônia, ansiedade; datas comemorativas; alimentação social e elaboração de receitas em laboratório, mostrando que é fácil e prático comer melhor.

Todos os conteúdos foram abordados através de palestras, dinâmicas e conversas em grupos. No primeiro encontro, as participantes responderam a três questionários: Questionário de Frequência Alimentar, Diário Alimentar e Rastreamento Metabólico. Para o rastreamento metabólico, foi aplicado um questionário do Instituto Brasileiro de Nutrição Funcional (IBNF), no qual foram analisados os sinais e sintomas que são classificados de acordo com a tabela de escala de pontos como mostra a Tabela 1.

Tabela 1. Escala de pontuação do Rastreamento Metabólico pelo IBNF.

Escala de pontos

\begin{tabular}{cc}
\hline 0 & Nunca ou quase nunca teve sintoma \\
1 & Ocasionalmente teve, efeito não foi severo \\
2 & Ocasionalmente teve, efeito foi severo \\
3 & Frequentemente teve, efeito não foi severo \\
4 & Frequentemente teve, efeito foi severo \\
\hline
\end{tabular}

Fonte: IBNF, 2016. 
Questionário de Rastreamento Metabólico (QRM) foi elaborado pelo Instituto Brasileiro de Nutrição Funcional, através dele é possível investigar variados sinais e sintomas onde o próprio paciente dará sua pontuação, avaliando de acordo com o que está sentindo nos últimos 30 dias, última semana ou últimas 48 horas, e no final, esses pontos são somados e utilizados como parâmetro para iniciar um rastreamento de prováveis deficiências nutricionais, hipersensibilidades, intolerâncias e alergias alimentares (RIBEIRO et al., 2017). A interpretação do resultado da soma de todos os sinais e sintomas é interpretado pelo IBNF (Tabela 2).

Tabela 2. Resultados interpretados através da pontuação do RM pelo IBNF.

\begin{tabular}{|c|c|}
\hline INTE & 10 DUS \\
\hline $\begin{array}{l}20 \text { pontos } \\
>30 \text { pontos } \\
>40 \text { pontos } \\
>100 \\
\text { Pontos }\end{array}$ & $\begin{array}{l}\text { Pessoas mais saudáveis, com menor chance de terem hipersensibilidade } \\
\text { Indicativo de existência de hipersensibilidade } \\
\text { Absoluta certeza de hipersensibilidade } \\
\text { Pessoa com a saúde muito ruim- alta dificuldade para executar tarefas diárias, pode } \\
\text { estar associado à presença de outras doenças crônicas } \\
\text { degenerativas. }\end{array}$ \\
\hline
\end{tabular}

Fonte: IBNF, 2016.

Após os atendimentos individualizados, foram elaborados planos alimentares individuais, os quais continham orientações alimentares para auxílio no processo de emagrecimento, mantendo os hábitos individuais das participantes.

Os dados foram tabulados por uma planilha no Microsoft Office Excel, ferramenta onde foi realizada a comparação do rastreamento metabólico do primeiro encontro e do último encontro, com objetivo de observar se houve nos sinais e sintomas a adequação da alimentação nas participantes.

\section{RESULTADO E DISCUSSÃO}

Participaram da pesquisa 8 (oito) mulheres. Foram entregues a todas as participantes os QRM, os quais foram respondidos no primeiro e no décimo encontro.

Através dos resultados das participantes, nos dados obtidos relacionados a sintomas da cabeça, olhos, ouvidos, nariz, boca e garganta, obteve-se um resultado de $54,1 \%$ de melhora comparando o questionário do primeiro e último encontro, através da somatória desses sinais e sintomas podem ser visualizadas na Tabela 3. De acordo com o estudo de Casanova-Rosado et al., (2006), existem sinais e sintomas relacionados a disfunção temporomandibular que causam dor. Com maior significância, as causas foram ansiedade, bruxismo, mordida unilateral e estresse. Um estudo realizado por Maltsman-Tseikhin et al., (2007) discute que a síndrome da ardência da boca pode estar relacionada a fatores locais e psicológicos, incluindo deficiências nutricionais, alterações hormonais, infecções orais, xerostomia, reações de hipersensibilidade e uso de medicamentos e doenças, como o diabetes mellitus. Os fatores mencionados pelos outros autores podem estar relacionados com os sinais e sintomas dos participantes avaliados. Para a comprovação desse dado, é necessário que o atendimento seja específico.

Outra variável com maior significância é relacionada ao sinais e sintomas que envolvem energia, mente e emoções, quando verificamos a pontuação das participantes comparando a pontuação do primeiro e último encontro, verificou-se $44,2 \%$ de melhora dos sintomas. Os resultados comparados tiveram diferenças significativas, mostrando diminuição desses sintomas após as dez semanas de 
acompanhamento nutricional (Tabela 3). De acordo com Carnauba, Baptistella e Paschoal (2018) e Naves, et al.,(2014), um dos princípios da Nutrição Funcional é a energia. Através dela podemos avaliar o metabolismo energético e a função da mitocôndria. O estresse emocional, alta ingestão de frituras, alto consumo de bebidas alcoólicas, exercícios físicos exaustivos, medicamentos, exposição a poluentes e toxinas ambientais, etc., são os fatores que podem promover o aumento do estresse oxidativo, levando a importantes desequilíbrios funcionais nas células e podem aumentar o risco de diversas doenças. Por meio do questionário e conversas com as participantes, pode-se perceber que os sintomas relacionados à energia, mente e emoções foram os itens que mais têm afetado o dia a dia e o desempenho de atividades diárias.

Tabela 3. Somatório dos Sinais e Sintomas apresentados pelos participantes no $1^{\circ}$ e $10^{\circ}$ encontro de acordo com o Questionário de Rastreamento Metabólico

\begin{tabular}{|c|c|c|c|c|c|}
\hline Participante & $\begin{array}{c}19 \\
\text { Encontro }\end{array}$ & $\begin{array}{c}10^{\circ} \\
\text { Encontro }\end{array}$ & Participante & $\begin{array}{c}1 \% \\
\text { Encontro }\end{array}$ & $\begin{array}{c}10^{\circ} \\
\text { Encontro }\end{array}$ \\
\hline \multicolumn{3}{|c|}{$\begin{array}{l}\text { Sinais e sintomas relacionados a cabeça, } \\
\text { olhos, ouvidos, nariz, boca e garganta. }\end{array}$} & \multicolumn{3}{|c|}{$\begin{array}{c}\text { Sinais e sintomas relacionados ao coração e } \\
\text { pulmão }\end{array}$} \\
\hline 1 & 21 & 1 & 1 & 3 & 1 \\
\hline 2 & 4 & 7 & 2 & 2 & 1 \\
\hline 3 & 28 & 17 & 3 & 14 & 8 \\
\hline 4 & 49 & 31 & 4 & 24 & 20 \\
\hline 5 & 30 & 20 & 5 & 2 & 3 \\
\hline 6 & 11 & 4 & 6 & 1 & 0 \\
\hline 7 & 10 & 4 & 7 & 2 & 2 \\
\hline 8 & 34 & 2 & 8 & 1 & 1 \\
\hline Total & 187 & 86 & Total & 46 & 36 \\
\hline \multicolumn{3}{|c|}{$\begin{array}{c}\text { Sinais e sintomas relacionados à energia, } \\
\text { mente, emoções }\end{array}$} & \multicolumn{3}{|c|}{$\begin{array}{c}\text { Sinais e sintomas relacionados ao trato } \\
\text { digestivo. }\end{array}$} \\
\hline 1 & 43 & 1 & 1 & 21 & 0 \\
\hline 2 & 16 & 3 & 2 & 6 & 4 \\
\hline 3 & 46 & 33 & 3 & 8 & 7 \\
\hline 4 & 52 & 44 & 4 & 9 & 3 \\
\hline 5 & 30 & 24 & 5 & 16 & 11 \\
\hline 6 & 3 & 2 & 6 & 3 & 2 \\
\hline 7 & 34 & 8 & 7 & 8 & 2 \\
\hline 8 & 25 & 7 & 8 & 7 & 4 \\
\hline Total & 219 & 122 & Total & 78 & 33 \\
\hline \multicolumn{3}{|c|}{$\begin{array}{c}\text { Sinais e sintomas relacionados a pele e } \\
\text { articulações }\end{array}$} & \multicolumn{3}{|c|}{$\begin{array}{l}\text { Sinais e sintomas relacionados a outros } \\
\text { sintomas }\end{array}$} \\
\hline 1 & 22 & 3 & 1 & 3 & 0 \\
\hline 2 & 7 & 4 & 2 & 5 & 2 \\
\hline 3 & 16 & 11 & 3 & 8 & 2 \\
\hline 4 & 27 & 18 & 4 & 5 & 0 \\
\hline 5 & 16 & 13 & 5 & 1 & 1 \\
\hline 6 & 6 & 4 & 6 & 0 & 0 \\
\hline 7 & 13 & 3 & 7 & 2 & 0 \\
\hline 8 & 17 & 4 & 8 & 4 & 0 \\
\hline Total & 124 & 60 & Total & 28 & 5 \\
\hline
\end{tabular}

No item que se refere a sinais e sintomas relacionados a pele e articulações, analisou- se uma melhora dos sintomas de $51,6 \%$ quando comparado ao questionário respondido no primeiro e décimo encontro (Tabela 3). Um estudo feito por López (2016), observou que as vitaminas têm vários benefícios para o nosso organismo. A ingestão inadequada de vitaminas pode levar a fraqueza capilar, envelhecimento 
prematuro, pouco crescimento e/ou decadência e problemas ósseos e cansaço. Juntamente às vitaminas, sabemos que o consumo adequado de água é essencial para uma boa hidratação da pele e para a lubrificação das articulações. De acordo com os dados obtidos, foi possível perceber a diminuição dos sinais e sintomas quando as participantes foram desafiadas a montar um prato colorido e saudável e beber no mínimo seis copos de água por dia.

Quando comparados sinais e sintomas relacionados ao coração e pulmão, verificou- se $21,7 \%$ de melhora dos sintomas (Tabela 3 ). A população tem procurado praticidade e não qualidade dos alimentos, com isso, o consumo em excesso de alimentos que contém sódio tem sido um fator importante no desencadeamento de doenças como hipertensão arterial. De acordo com Dutra e Silva (2019), a palpitação, dificuldade para respirar, e dor no peito estão associados a hipertensão arterial. Ao avaliar a pontuação das participantes, se faz necessário uma investigação desses sintomas, uma vez que nenhuma relatou ter hipertensão arterial, somente os sintomas citados.

Já nos sintomas que envolvem o trato digestivo, pode-se observar que houve melhora de 57,6\% dos sintomas (Tabela 3). Fagundes (2010), aplicou o QRM para avaliar a disbiose intestinal e teve como resultado que sintomas relacionados ao trato digestivo representaram uma das queixas mais comuns, constatando que $31 \%$ dos participantes obtiveram mais que 10 pontos no bloco relacionado ao trato GI. No presente estudo, foi possível verificar que a maioria dos participantes obtiveram pontuação inferior a 10 pontos, sendo um item de pouca relevância.

No tópico de outros sintomas que incluem itens como: frequentemente doente, frequentemente ou urgência de urinar, coceira genital ou corrimento e edema nos pés, pernas, mãos e braços (Tabela 3), pode-se observar que houve $82,1 \%$ de melhora dos sinais e sintomas e nenhuma participante marcou pontuação superior a 20 pontos, sem indicativo de ter hipersensibilidades. Observando e comparando a soma total de todos os itens mencionados, das oito participantes que responderam o questionário no primeiro e décimo encontro, podemos observar uma melhora de 48,29 \% do total dos sinais e sintomas.

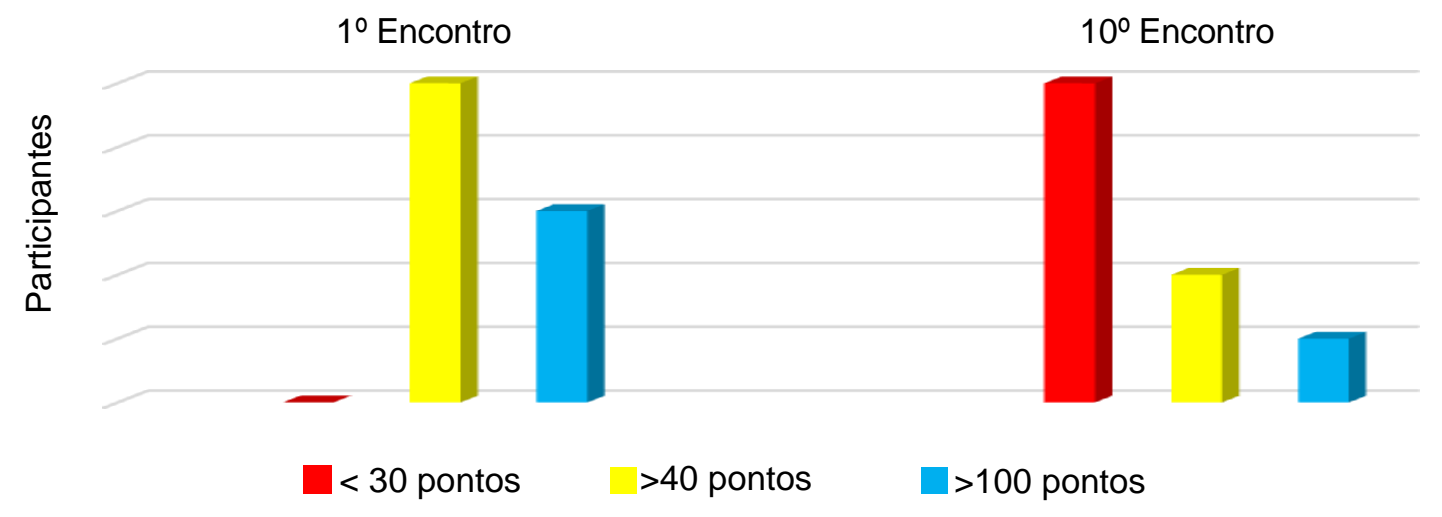

Figura 1. Somatória total dos questionários

De acordo com a interpretação dos resultados mostrado na Tabela 2, podese observar que no primeiro encontro a somatória total de 5 participantes foi $>40$ pontos que é indicativos de absoluta certeza de hipersensibilidade e de 3 participantes foi >100 pontos, indicativo de que a pessoa está com a saúde muito ruim. Já no décimo encontro podemos verificar que a somatória total de 5 participantes foi $<30$ pontos indicando que a pessoa está com menos sinais e sintomas, de 2 pessoas foram $>40$ 
pontos, que é indicativo de absoluta certeza de hipersensibilidade e de 1 pessoa foi >100 pontos, indicativo de que a pessoa está com a saúde muito ruim (Figura 1).

\section{CONSIDERAÇÕES FINAIS}

O estudo contou com oito participantes assíduas do grupo de emagrecimento, frequentando os encontros uma vez por semana durante dez semanas. Após esse acompanhamento e aplicação do questionário de rastreamento metabólico, pode-se observar que houve melhora significativa com média de $48,29 \%$ de melhora nos sinais e sintomas das participantes, submetidas a um acompanhamento nutricional. Deste modo, é notório que o acompanhamento nutricional é imprescindível para promoção e prevenção de doenças.

\section{REFERÊNCIAS}

CARNAUBA, Renata Alves; BAPTISTELLA, Ana Beatriz; PASCHOAL, Valéria. Nutrição clínica funcional: uma visão integrativa do paciente. Instituto VP de Pesquisa. 2018. Disponível em: http://docs.bvsalud.org/biblioref/2018/04/882170/rdt_v23n1_28-32.pdf. Acesso em 15 de mar.2019.

CASANOVA-ROSADO J. F; MEDINA-SOLÍS, C. E; VALLEJOS-SÁNCHEZ, A. A; CASANOVA-ROSADO, A. J; HERNÁNDEZ-PRADO, B; ÁVILA-BURGOS, L. Prevalence and associated factors for temporomandibular disorders in a group of Mexican adolescents and youth adults. Clin Oral Invest. 2006. Disponível em: https://www.ncbi.nlm.nih.gov/pubmed/16311741. Acesso em: 25 fev. 2019.

DUTRA, Norivan Lustosa Lisboa; SILVA, Thaise Liara da (Org.). Mulheres Mil na Prevenção da Obesidade, Hipertensão e Diabetes. 2013. 1. ed. - Goiânia: IF Goiano. 84 p. ISBN: 978-85-65871-03-7. Disponível em: <https://suap.ifgoiano.edu.br/media/documentos/arquivos/LIVRO_MULHERES_MIL. pdf>. Acesso em: 02 jun. 2019.

FAGUNDES, G. E. Prevalência de sinais e sintomas de disbiose intestinal: sintomas de disbiose intestinal em estudantes do curso de nutrição da universidade em estudantes do curso de nutrição da universidade do extremo sul do extremo sul catarinense. $2010 . \quad$ Disponível em https://docs.google.com/viewerng/viewer?url=http://www.rbone.com.br/index.php/rbo ne/artic le/viewFile/790/592. Acesso em: 03 abr. 2019

FRANÇA C. L. P; BARBOSA M. B. D; MUdESTO A. P. L.; ALVES E. D. Contribuições da psicologia e da nutrição para a mudança do comportamento alimentar. 2012. Disponível em:

http://www.scielo.br/scielo.php?script=sci_arttext\&pid=S1413- 294X2012000200019. Acesso em: 02 marc. 2019.

GALDINO, J. J.et al. Questionário de rastreamento metabólico voltado a disbiose intestinal em profissionais de enfermagem. 2016. Disponível em: 
http://www.rbone.com.br/index.php/rbone/article/view/422. Acesso em: 25 fev. 2019. Acesso em: 25 de fev.2019.

GIL, A. C. Métodos e técnicas de pesquisa social. 6ª edição. São Paulo, Atlas, 2008.

LÓPEZ, L. E. M. Seguridad alimentaria y nutricional: Causas y consecuencias de la falta de vitaminas en la alimentación diaria y su impacto en la salud humana. 2016. Disponível em: http://repositorio.unan.edu.ni/3778/1/11051.pdf. Acesso em: 03 de maio.2019.

MALTSMAN-TSEIKHIN A, MORICCA P, NIV D. Burning mouth syndrome: will better understanding yield better management? Pain Pract 2007 Jun;7(2):151-62.

MARTINEZ, S. A nutrição e a alimentação como pilares dos programas de promoção da saúde e qualidade de vida nas organizações. 2011. Disponível em: http://bvsms.saude.gov.br/bvs/artigos/mundo_saude/nutricao_alimentacao_pilares_p rogramas_promocao.pdf. Acesso em: 31 de maio.2019.

NAVES, A; PASCHOAL, V. E; PASCHOAL, V; NAVES, A. F. Nutrição clínica funcional: dos princípios à prática clínica. São Paulo: VP Editora; 2014. Disponível em: http://docs.bvsalud.org/biblioref/2018/04/882170/rdt_v23n1_28-32.pdf. Acesso em: 31 de março.2019.

RIBEIRO, P. V. M. et al. Evolução do estado nutricional e sintomatologia intestinal e extra intestinal em pacientes com doença celíaca e sensibilidade ao glúten não celíaca submetidos à orientação dietética especializada. Rev. Nutr. [online]. 2017, vol.30, n.1, pp.57-67. Disponível em: http://www.scielo.br/scielo.php?script=sci_arttext\&pid=S1415- 52732017000100057. Acesso em: 31 de março. 2019.

RIBEIRO, S. M. L. O processo de avaliação nutricional. In: Tirapegui J, Ribeiro SML. Avaliação nutricional: teoria e prática. Rio de Janeiro: Guanabara-Koogan;

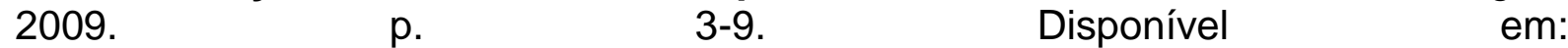
http://www.scielo.br/scielo.php?script=sci_arttext\&pid=S1415-790X2011000100015. Acesso em: 31 de março.2019

SILVA, G. M. F.; SILVA, T. K. R.; MELHEMC, A. R. F.; TORTORELLAD, C. C. S.; Avaliação nutricional, consumo alimentar e percepção de hábitos saudáveis entre escolares de 10 a 14 anos. 2018. Disponível em:

http://seer.uscs.edu.br/index.php/revista ciencias saude/article/viewFile/5215/pdf. Acesso em 28 de fev.2019.

TIRAPEGUI, J.; RIBEIRO, S. M. L.; Avaliação nutricional: teoria e prática. São Paulo, Guanabara Koogan, 2011.

VAZ, D. S. S; BENNEMANN, R. M. COMPORTAMENTO ALIMENTAR E HÁBITO ALIMENTAR: UMA REVISÃO. REVISTA UNINGÁ REVIEW, [S.I.], v. 20, n. 1, jan. 
2018. ISSN 2178-2571. Disponível em:

http://revista.uninga.br/index.php/uningareviews/article/view/1557. Acesso em: 01 de março. 2019. 